\title{
Reactivation of tuberculosis in cancer patients following administration of immune checkpoint inhibitors: current evidence and clinical practice recommendations
}

\author{
Amalia Anastasopoulou ${ }^{1 \dagger}$, Dimitrios C. Ziogas ${ }^{1 \dagger}$, Michael Samarkos ${ }^{1}$, John M. Kirkwood² and Helen Gogas ${ }^{1 *}$
}

\begin{abstract}
Immune checkpoint inhibitors (ICBs) have revolutionized cancer treatment producing remarkable and durable responses for a range of malignancies. However, the additional modulation of immune response by ICBs may rarely cause immune-related infectious complications, including re-activation of latent tuberculosis infection (LTBC) with detrimental effects on those patients' outcome. Here, we present two "real-world" melanoma cases that were treated in our department with blockade of PD-1/PD-L1 and developed active Mycobacterium tuberculosis (MTB) during immunotherapy. In view of these cases, we review the literature for ICB-associated MTB reactivation and discuss our considerations about the possible interactions of immunotherapy and the underlying co-existent mycobacterial infection. Based on the current evidence from preclinical findings prior to this experience, we raise questions regarding cancer patients who are at higher risk for developing MTB infection, whether ICB-treated patients should be considered immunocompromised, and how they should be managed for latent and/or active tuberculosis. Aside from the wellestablished clinical benefit of immunotherapy, the blockade of PD-1/PD-L1 axis may concurrently disrupt the immune control of specific opportunistic infections such as tuberculosis that should be carefully and expectantly managed in order to avoid compromising the outcome of cancer treatment and the affected patient's survival.
\end{abstract}

Keywords: Immunotherapy, Cancer, Immune checkpoint inhibitors, Tuberculosis

\section{Introduction}

The development of immune checkpoint inhibitors (ICBs) has radically changed the way that numerous cancers are treated. After the initial approval of ipilimumab (a monoclonal antibody blocking CTLA-4) for the treatment of metastatic melanoma, five more antibodies that target PD1/PD-L1 pathways, including nivolumab and pembrolizumab (against PD-1) and atezolizumab, avelumab, and durvalumab (against PD-L1), have been licensed and introduced into the therapeutic algorithms for various malignancies in first and later lines of treatment, as well as in the neoadjuvant and adjuvant settings [1]. Cancer cells are able to evade host immune surveillance and escape tumor

\footnotetext{
* Correspondence: helgogas@gmail.com

${ }^{\dagger}$ Amalia Anastasopoulou and Dimitrios C. Ziogas contributed equally to this work. ${ }^{1}$ First Department of Medicine, Laiko General Hospital, National and Kapodistrian University of Athens School of Medicine, 11527 Athens, Greece Full list of author information is available at the end of the article
}

neutralization by inhibiting PD-1 targeted cancer-specific $\mathrm{T}$ cells via overexpression of PD-L1 [2]. The monoclonal antibodies prevents the binding of PD-1 to its ligand PD$\mathrm{L} 1$, restores the $\mathrm{T}$ cell-mediated cytotoxicity and allows the immune natural defense to fight against cancer with significant clinical benefits [3]. However, the immune stimulation triggered by these drugs may lead to severe and even life-threatening, albeit infrequent, immune-related adverse events (irAEs) involving almost every organ $[4,5]$. Current guidelines on the management of irAEs recommend the prompt administration of high-dose corticosteroids and if toxicity persists, further immunosuppression with steroid-sparing regimens (e.g. anti-tumor necrosis factor-alpha (TNF-a) agents or mycophenolate mofetil) [6].

In this context, the additional modulation of immune response due to the cancer itself, due to the administration of ICPIs or the supplementary medications (e.g. 
steroids or anti-TNF agents) for overcoming irAEs may unmask chronic underlying or opportunistic infections and rarely cause some serious infectious complications such as varicella-zoster virus infection, cytomegalovirusassociated enterocolitis, pulmonary aspergillosis, pneumocystis pneumonia and reactivation of latent tuberculosis with detrimental, in some cases, effects on cancer treatment outcome and patient's survival $[7,8]$. The overall incidence of these serious immune-related infections in 740 patients with metastatic melanoma receiving ICBs was estimated to be $7.3 \%$ in a recent review, where infectious complications were detected mainly in patients who required corticosteroids and/or TNF-a inhibitors [9].

Given the high incidence of Mycobacterium tuberculosis (MTB) infection worldwide and the poor prognosis of MTB reactivation, a renewed interest was developed to recognize individuals at high risk that should be screened for early detection of latent tuberculosis and treated to prevent active disease [10,11]. The Center for Disease Control and Prevention (CDC), the World Health Organization (WHO), and the US Preventive Services Task Force (USPSTF) agree that the risk of exposure to MTB is higher: a) in patients living or working in endemic countries (e.g. East Asia and Central America) and $b$ ) in patients living in large group settings (e.g. homeless or military shelters and prisons). In most patients infected with MTB, the disease remains clinically asymptomatic and inactive, however, in $5-10 \%$ of them, the infection will reactivate at some point during their lifetime with a baseline risk between 6 and 20 per 100 , 000 person-years [12]. After that, the risk of reactivation depends on the specific type of immunosuppression [11, 13]. Compared to the general population, this risk is greater among solid organ transplant recipients (15xfold) [14] and stem cell transplant recipients (8-12xfold) [15], followed by patients treated with anti-TNF medications (5-7xfold) [16-19], while in patients with HIV infection, it reaches 50 times higher and causes up to $25 \%$ of deaths among patients [20]. Other host factors that may increase the susceptibility to develop active tuberculosis include older age ( $>60$ years), prior tuberculosis history, chronic obstructive pulmonary disease, heavy smoking or increased alcohol consumption, diabetes mellitus or end-stage renal disease and for these patients screening is also recommended [13, 21-23]. Cancer has been recognized as an independent risk factor for developing active MTB infection since the 1970s, however this risk widely varies among cancer types, is differentially affected by modern therapies (targeted agents and monoclonal antibodies) and remains to be precisely quantified.

In this study, we present two melanoma patients who developed active tuberculosis during their treatment with PD-1/PD-L1 blockade in our department. In view of these two cases, we review the literature from the preclinical data on the immune-mediated interactions of PD-1/PD-L1 inhibition and co-existent tuberculosis, and published clinical reports with ICB-associated tuberculosis. Integrating the current evidence with our institutional experience, we address questions about which cancer patients are at higher risk for MTB infection, whether ICB-treated cases should be still considered immunocompromised, and how they should be managed for latent or active tuberculosis.

\section{Case 1}

A 76-year-old Greek woman was diagnosed with a cutaneous melanoma lesion of her left lower leg in August 2009 (Fig. 1). Her comorbidities included smoking of 45 pack*- $^{*}$ years, hypertension, dyslipidemia, coronary artery disease and osteopenia. She underwent a radical resection of the tumour, but the sentinel lymph node was grossly infiltrated (stage IIIb, T3aN1aM0), and she received interferon (IFN) $20,000 \mathrm{iu} / \mathrm{m}^{2}$ every day during December 2009, according to contemporary recommendations. She remained disease free until July 2017 when she developed a new cutaneous lesion of her left calf (M1a, stage IV). PET/CT scanning did not show other distant metastasis. For her metastatic recurrent melanoma, the patient enrolled in a clinical trial (ClinicalTrials.gov ID: NCT03068455) and was randomized to receive monotherapy with nivolumab $240 \mathrm{mg}$ every 2 weeks versus the combination of nivolumab with ipilimumab $1 \mathrm{mg} / \mathrm{kg}$ every 3 weeks. Due to her smoking history, she was under regular follow-up by pulmonologist and had a negative tuberculin skin test (TST) on March 2017 but the trial protocol did not require LTBC screening before the initiation of immunotherapy. In January 2018, after 8 doses of immunotherapy, she presented diarrhea grade 2 and started methylprednisolone $16 \mathrm{mg}$ po twice daily with a slow taper (over $4-6$ weeks). After a short-term improvement of her diarrhea to grade 1 , her symptoms worsened again and a colonoscopy was performed. The endoscopic examination revealed grade 3 colitis with multiple ulcerative mucosal lesions. Therefore, immunotherapy was permanently discontinued and the dose of methylprednisolone was increased to $32 \mathrm{mg}$ daily and intravenous (iv) infliximab administered at a dose of $5 \mathrm{mg} / \mathrm{kg}$. After three doses of infliximab her colitis improved (to grade 1) and steroid taper was resumed. Two weeks later, the patient was admitted to our hospital with fever up to $38^{\circ} \mathrm{C}$, fatigue and weight loss. Physical examination suggested a lower respiratory tract infection. Laboratory tests revealed neutrophils: $6700 / \mu \mathrm{L}$, hemoglobin: 10.5 g/dL, platelet count: $129,000 / \mu \mathrm{L}$ and elevated $C$ reactive protein $(C R P=147.6 \mathrm{mg} / \mathrm{L})$. The patient was empirically treated with iv tazobactam/piperacillin $4.5 \mathrm{mg} 4$ times daily. Computed tomography (CT) scanning of the chest showed interval development of ground glass and centrilobular nodular opacities predominantly in the right lung 


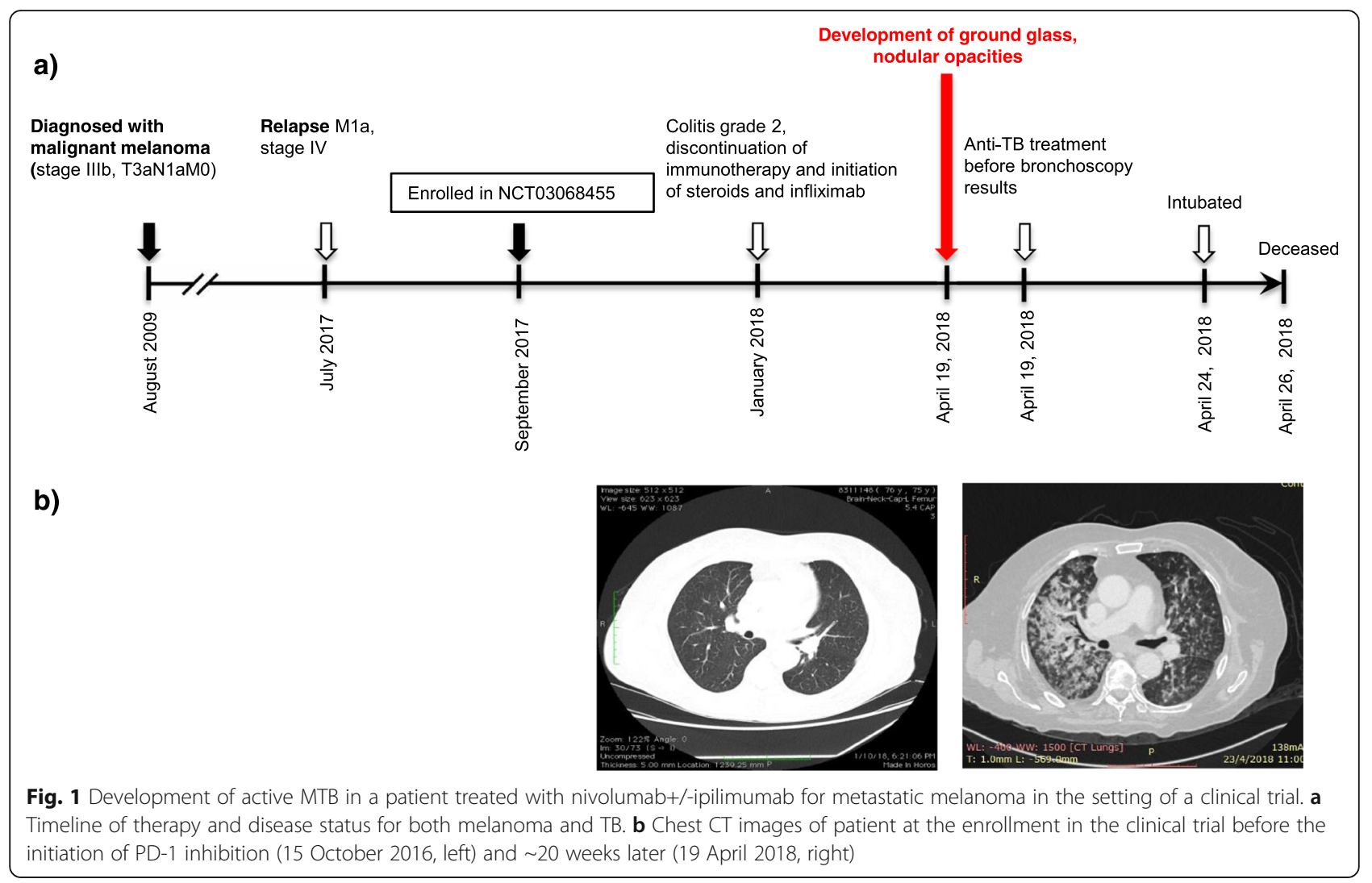

(Fig. 1). These imaging findings were not present in a prior CT scan 2 months before. On April 19, 2018, the patient underwent bronchoscopy and was started on anti-tuberculosis medication with rifampin $600 \mathrm{mg} /$ day, isoniazid 300 $\mathrm{mg} /$ day and ethambutol $1200 \mathrm{mg} /$ day and pyrazinamide $2000 \mathrm{mg} /$ day as well as anti-pneumocystis treatment with trimethoprim-sulfamethoxazole $20 \mathrm{mg} / \mathrm{kg} /$ day. The polymerase chain reaction (PCR) of bronchoalveolar lavage (BAL) was positive for MTB complex. Despite immediate treatment, her respiratory function was progressively worsened and the patient was moved to intensive care unit. She was intubated but remained persistently febrile and hypotensive requiring vasopressor medications. The patient passed away 2 days later and subsequently the BAL culture grew MTB with no resistance to anti-tuberculosis treatment, according to susceptibility testing.

\section{Case 2}

An 85-year-old Greek man was diagnosed with melanoma of the right parotid nodes confirmed by positive fine needle aspiration on December 2012 (Fig. 2). His medical history included hypertension, dyslipidemia, glaucoma and benign prostate hyperplasia. The patient underwent a total parotidectomy and regional lymph node dissection as well as a tonsil biopsy. Both right parotid gland and dissected lymph nodes were infiltrated by melanoma, while biopsy of tonsil was negative. No primary skin lesion was recognized and subsequent staging scans were also negative for residual disease (stage IIIb, TxN1bM0). Therefore, he received prophylaxis with high dose IFN $20000 \mathrm{iu} / \mathrm{m}^{2}$ every day and he was followed-up until June 2018. At this time, he underwent a chest CT because of persistent cough, which revealed multiple lymph nodes and a mediastinal soft tissue mass (M1b, stage IV) (Fig. 2). In the context of a clinical trial (ClinicalTrials.gov ID: NCT03273153), he started a combination with atezolizumab $840 \mathrm{mg}$ every 3 weeks and MEK inhibitor cobimetinib $60 \mathrm{mg}$ every day for his metastatic BRAFV600 wild-type melanoma. The patient was not tested for LTBC before starting antimelanoma treatment, because he had no known risk factors for MTB reactivation and the clinical trial protocol did not require it. After receiving the therapeutic combination for approximately 5 months, including a temporary interruption of cobimetinib because of grade 3 rash, the patient presented with symptoms of lower respiratory tract infection grade 3 on November 2018. No new imaging findings by X-ray were recognized at that time. Cobimetinib was interrupted again and the patient received a course of iv tazobactam/piperacillin $4.5 \mathrm{mg}$ QID and levofloxacin $500 \mathrm{mg}$ every day. During the next 3 months, the patient had two more episodes of fever grade 2, during which he was hospitalized and received broad-spectrum antibiotics for 1 week in total each time. 


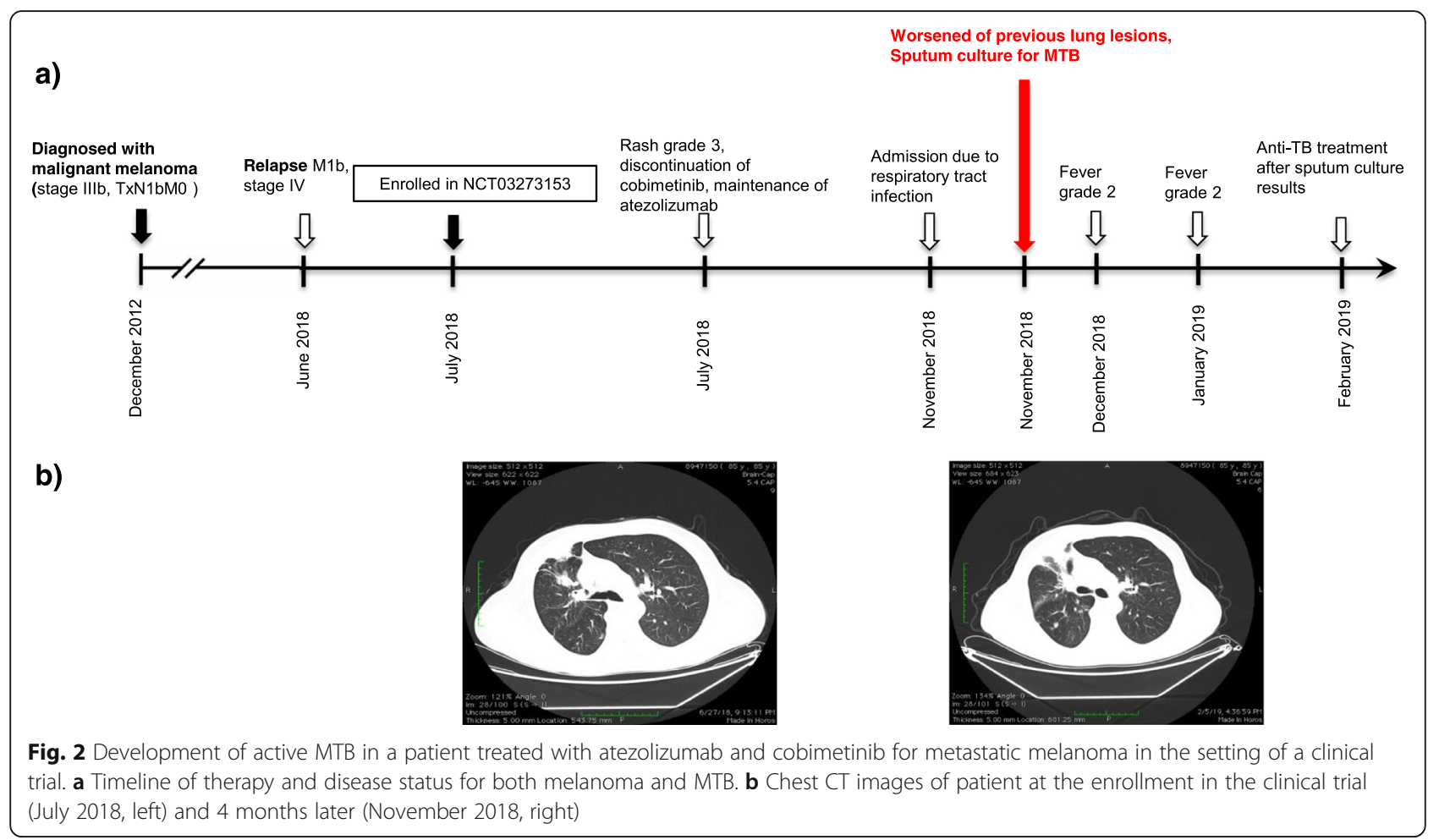

Throughout this period, cobimetinib was temporarily interrupted, but immunotherapy with atezolizumab was continued without complications. Although acid-fast bacilli stain of sputum was negative, a sputum culture taken in his last hospitalization grew M. tuberculosis. Susceptibility testing showed susceptibility to all antimycobacterial agents. On February 2019, he initiated a 3drug regimen, including isoniazid $300 \mathrm{mg} /$ day, rifampin $600 \mathrm{mg} /$ day and pyrazinamide $1500 \mathrm{mg} /$ day. Currently, he continues anti-tuberculosis medication with good tolerability, whereas anti-melanoma combination is still withheld up to resolution of MTB imaging lesions.

\section{Preclinical data in mice and human cells}

Although T-helper type 1 CD4+ cells (Th1) are required for control of mycobacterial infections, the enhanced CD4 activity in the absence of PD-1 surveillance exacerbates tuberculosis in mouse models. This status of $\mathrm{T}$ cell exhaustion arises from the sustained activation with absence of inhibitory receptors and prevents optimal control of infection and tumors [24]. In fact, PD-1 knockout (PD-1-/-) mice are more susceptible to MTB mortality, developing large necrotic lesions with high bacterial loads and succumbing faster than even $\mathrm{T}$ cell-deficient mice [25-27]. The inability of PD-1-/- mice to control mycobacterial infection is attributed to increased Th1-mediated responses and overproduction of interferon-gamma (IFN- $\gamma$ ) [26]. Sakai et al. pointed out that activated PD-1/PD-L1 signaling suppresses the accumulation of parenchymal
CD4+ $\mathrm{T}$ cells and limits IFN- $\gamma$ production, protecting mice from fatal exacerbated pulmonary mycobacterial infection [28]. Cancer cells and infectious agents may evade early immune responses with other PD-1/PD-L1 mediated mechanisms: i) promotion of PD-L1 expression on dendritic cells and enhanced induction of Treg cells [29, 30], ii) overexpression of PD-1 on NK cells, as detected in patients with multiple myeloma [31] or infected with MTB [32] or HIV [33]. A recent study by Cao et al. [34] found that co-stimulation by MTB and lung cancer antigen in mice may partially reverse the loss of $\mathrm{T}$-cell function via PD-1/PD-L1 pathway and prevent the rapid evolution of advanced lung cancer [34].

In human patients with active tuberculosis, PD-1 was increased on CD4+ T cells but not on CD8+ T cells compared to healthy controls $[35,36]$ while effective anti-tuberculosis treatment was associated with a downregulation of PD-1 on CD4+ T cells [36]. Similarly, the expressions of PD-1 and PD-L1 on monocytes from patients with active MTB were much higher compared to healthy controls, while phagocytosis and intracellular killing activity of macrophages increased significantly with PD-1/PDL1 blockade in vitro [35]. In a patient with Merkel cell carcinoma treated with nivolumab, IFN- $\gamma$-producing MTBspecific CD4+ T cells were detected in the blood months before the development of a tuberculoma [37]. Producing a scenario similar to immune reconstitution inflammatory syndrome (IRIS), the blockade of PD-1 axis boosts Th1mediated inflammatory responses and causes a worsening 
of damage to the MTB-infected tissue [38]. Currently, the PD-1/PD-L1 pathway is also being studied as a novel host-directed target in multidrug-resistant tuberculosis $[39,40]$.

\section{Literature search}

In order to identify other reported cases with ICB-associated MTB infection, we used the following terms for online search of PubMed: (1) terms suggestive of cancer (e.g., cancer, tumor, malignancy), (2) terms suggestive of immunotherapy (e.g., immune checkpoint inhibitors, PD-L1, PD-1, CTLA4, immunotherapy), (3) terms suggestive of tuberculosis (e.g., tuberculosis, TB, Mycobacterium tuberculosis). As restriction limits through the electronic search, we used English language and human-based studies. The full search strategy of the literature by the two independent reviewers, with the numbers of the records identified or excluded and the reasons of exclusions, is represented in Fig. 3, according to the PRISMA (Preferred Reporting Items for Systematic Reviews and Meta-analysis). A secondary expanded search was conducted by using Medical Subject Headings (MeSH) subjects and by hand-searching of reference lists from previous reviews to identify additional publications. For the $\mathrm{cu}-$ mulative presentation of findings among the identified case-reports, the $95 \% \mathrm{CIs}$ of proportions were estimated by modified Wald method.

\section{Current evidence on MTB reactivation after ICPI treatment}

We have identified ten reports that describe 12 cancer patients with active MTB infection after PD-1/PD-L1 blockade [37, 41-49]. Table 1 summarizes all these published cases with the addition of the two cases reported

Search strategy:

("cancer" or "tumor" or "neoplasm" or "malignancy" or "carcinoma") and ("immune checkpoint inhibitors" or "PD-L1" or "PD-1" or "CTLA4" or "immunotherapy") and ("tuberculosis" or "TB" or "Mycobacterium tuberculosis")

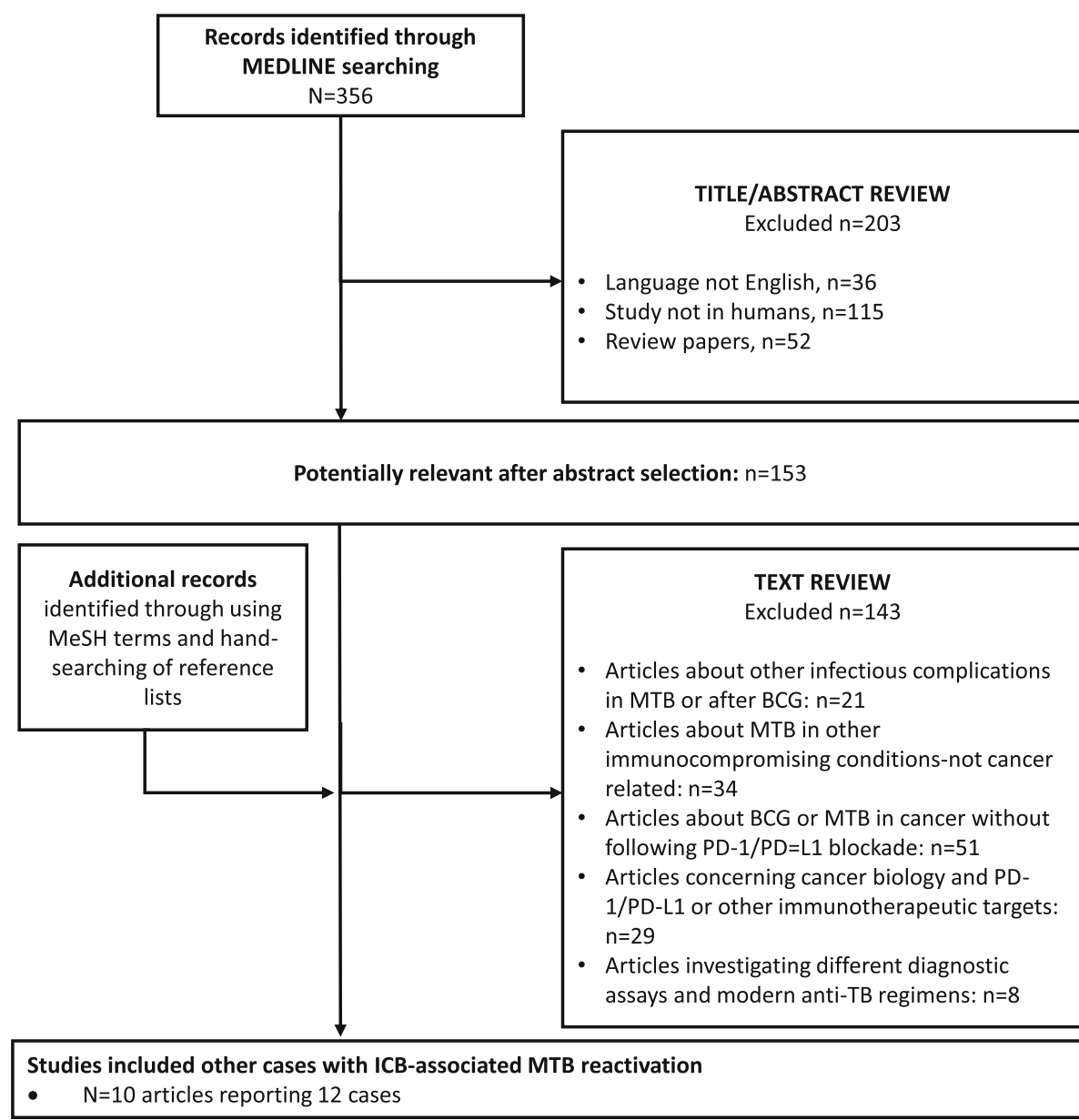

Fig. 3 Flow diagram of literature search strategy 
here. The reported patients were predominantly males (78.57, 95\%CI:51.68-93.16\%) and their age ranged from 49 to 87 years. Of 14 cases, 5 cases had advanced/metastatic non-small cell lung cancer (NSCLC) (35.71, 95\%CI:16.18-61.40\%), 5 had advanced/metastatic melanoma (35.71, 95\%CI:16.18-61.40\%), 2 had advanced/ metastatic head and neck squamous carcinoma (HNSC C) $(14.29,95 \% \mathrm{CI}: 2.76-41.19 \%), 1$ had metastatic Merkel carcinoma (7.14, 95\%CI: $<0.01-33.54 \%)$, and 1 Hodgkin lymphoma $(7.14,95 \% \mathrm{CI}:<0.01-33.54 \%)$. For PD-1/PDL1 inhibition nivolumab was used in 8 cases (57.14, 95\%CI:32.55-78.66\%), pembrolizumab in 5 cases (35.71, 95\%CI:16.18-61.40\%) and atezolizumab in the last case $(7.14,95 \% \mathrm{CI},<0.01-33.54 \%)$. There are several points of interest. Only two of 14 patients $(14.29,95 \% \mathrm{CI}, 2.76-$ 41.19\%) that developed active MTB infection had required steroids or infliximab for any irAEs. Apart from the first case presented here only the case reported by Chu et al. [43] had received prednisolone $1 \mathrm{mg} / \mathrm{kg}$ for 1 month. Thus, cancer and ICB immunotherapy should be considered the probable basis for susceptibility to MTB in these cases. In most cases the initial diagnosis at onset of MTB-associated illness was cancer progression, due to shared findings of weight loss and new lung infiltrates. In the reported cases, it was not clear whether tuberculosis was primary or secondary to reactivation of latent disease. In all cases with available time-to-event information, the symptoms and signs of MTB infection developed within 6 months of PD-1/PD-L1 inhibition, suggesting latent tuberculosis reactivation. However, none of the patients had undergone any testing for LTBC before the initiation of immunotherapy despite MTB mortality rate of $28.57 \%$ (95\%CI,11.34-55.03\%). Regarding the treatment of ICB-associated MTB, all patients received rifampicin-containing regimens and cancer immunotherapy was temporarily discontinued in 5 cases $(35.71,95 \% \mathrm{CI}, 16.18-61.40 \%)$ while maintained in 3 cases (21.43, 95\%CI,6.84-48.32\%).

\section{Clinical recommendations}

Based on the synthesis of current evidence and our experience, we below address some emerging issues regarding the incidence and management of tuberculosis in oncological patients, and suggest clinical practice recommendations.

\section{Recommendations for LTBC screening in cancer patients}

There is no clear recommendation whether cancer patients should be screened for LTBC and if positive, receive preventive chemoprophylaxis. According to 2018 WHO guidelines, cancer patients are not suggested for LTBC screening due to the lack of evidence [50], The USPSTF did not review evidence on screening of patients with any type of malignancy, since screening of these populations was already indicated prior to certain immunosuppressive medications, including chemotherapy or TNF-a inhibitors [51]. Current guidelines of the American Thoracic Society (ATS), the CDC and the Council of the Infectious Diseases Society of America (IDSA) recognize patients with leukemias and lymphomas, with head and neck or lung carcinoma as highrisk cases for MTB reactivation and subsequently recommend chemoprophylaxis when LTBC is documented in these groups $[10,52]$. These guidelines are derived from studies between 1950s and 1970s and are limited by the absence of observation time at the estimation of relative risk. The risk for developing MTB is different among cancer types and continues to change over time as newer therapeutic strategies are developed. Targeted monoclonal antibodies and hematopoietic stem cell transplantation modified drastically the management of hematologic malignancies and produced diverse patterns of immunosuppression compared to therapies prior to the 1970s [53] while for HNSCC and lung cancer, new radiation modalities have decreased local tissue damage [54]. According to the National Institute for Health and Care Excellence (NICE), patients with LTBC having a hematological malignancy, having chemotherapy for any cancer type or having a gastrectomy for gastric cancer are at increased risk of developing tuberculosis, however, NICE does not provide specific screening and treatment recommendations for these groups [55]. Using Danish nationwide medical databases, Simonsen et al. concluded that the risk for active tuberculosis among cancer patients was significantly higher compared to age/sexmatched healthy controls after adjustment for other comorbidities [56]. The highest risks were observed in cancers of the aerodigestive tract, tobacco-related cancers, and hematologic malignancies [56]. Recently, Cheng et al. performed a systematic review and meta-analysis to quantify the risk of active MTB infection in cancer patients, including 23 studies with more than 300,000 patients [11]. Despite the methodological limitations, this study showed that individuals with hematologic, HNSCC, and lung cancers had a higher rate of developing active MTB compared to those without cancer and would benefit from targeted LTBC screening and chemoprophylaxis [11]. More specifically, in the six studies from United States published after 1980, the incidence rate ratio (IRR) was 26 for hematologic malignancies, 16 for HNSCC, 9 for NSCLC, and 4 for breast and other solid tumors [11]. For HNSCC and lung carcinoma, this increased risk may be confounded by other independent risk factors such as alcohol usage or smoking [57]. Dobbler et al. conducted another meta-analysis including 13 studies with more than 920,000 patients to estimate further the IRR of tuberculosis for patients with solid and hematologic malignancies compared to the 


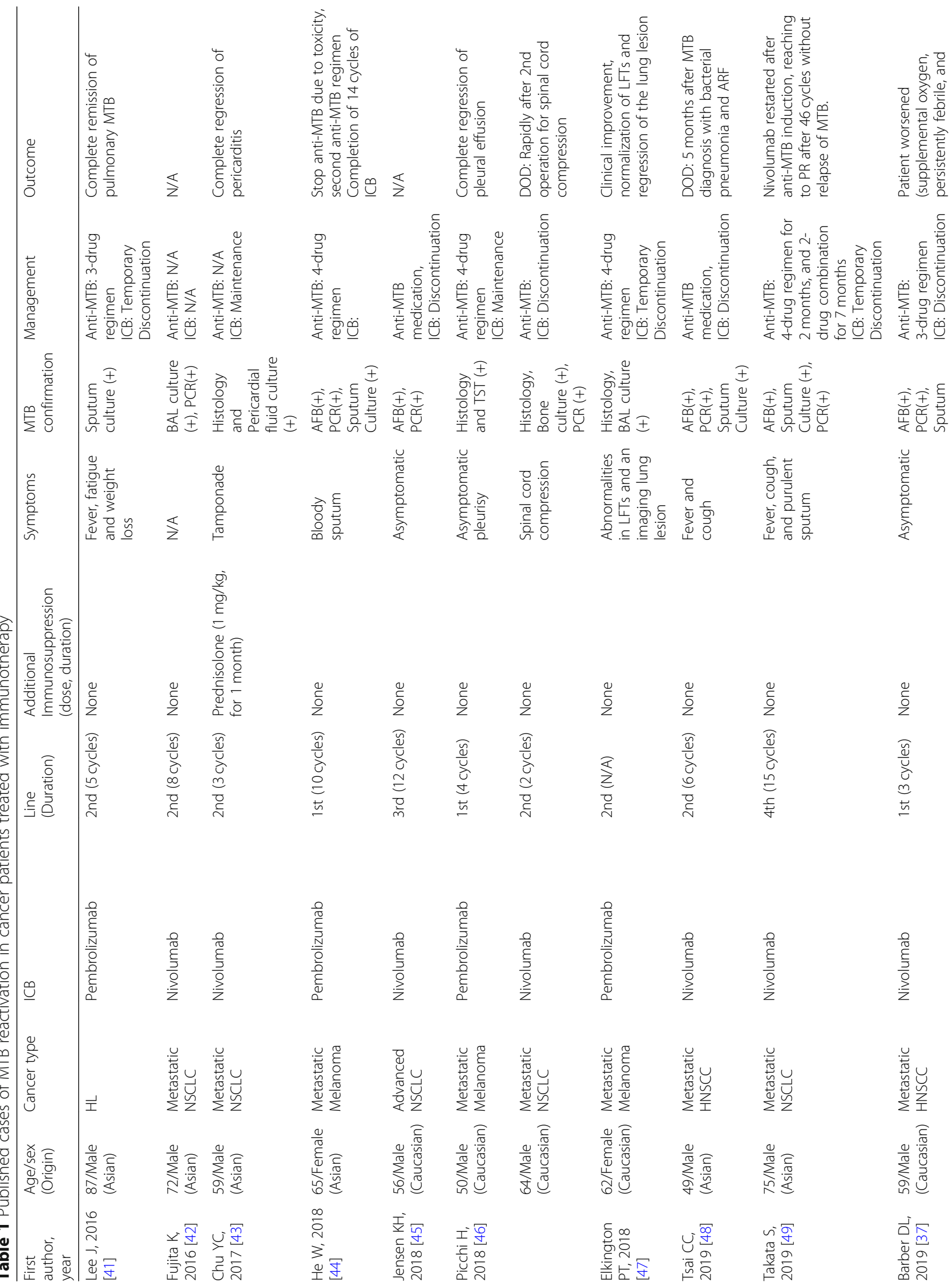



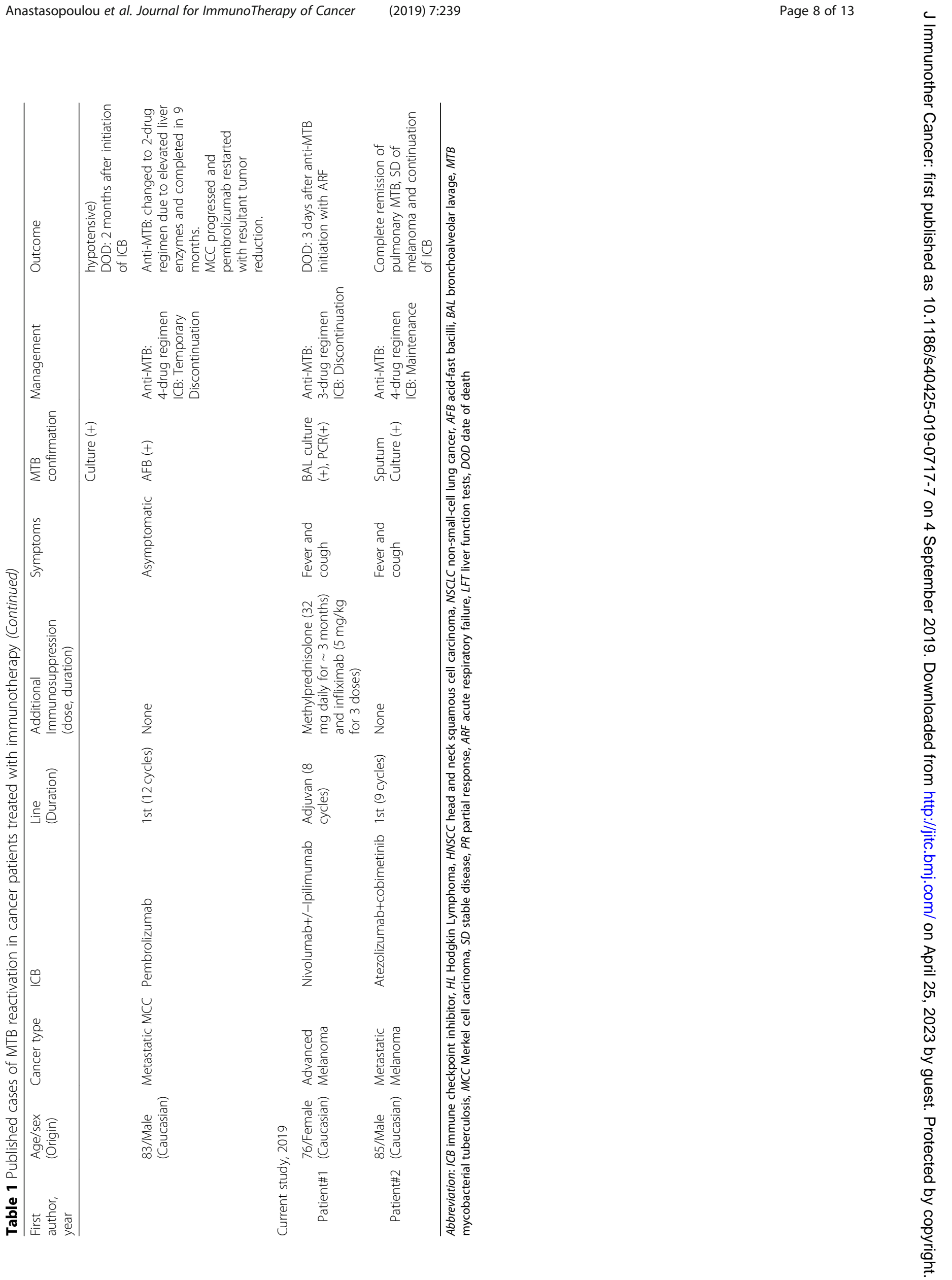
general population [10]. In this study, lung $(\mathrm{IRR}=6.14$; 95\%CI:1.97-19.20), gastric $(\mathrm{IRR}=2.63$, 95\%CI:1.96$3.52)$, breast (IRR $=2.17 ; 95 \% \mathrm{CI}: 1.98-2.38)$ and colon cancer (IRR $=2.00,95 \% \mathrm{CI}: 1.16-3.43)$, had a statistically significant greater IRR of developing MTB infection, instead of liver cancer that did not reach significance $(\mathrm{IRR}=2.02 ; 95 \% \mathrm{CI}: 0.83-4.91)$ [10]. Gastric cancer had not a remarkably different IRR from other solid cancers, although it is often treated with gastrectomy and characterized by malnutrition [58], an independent risk factor for tuberculosis [59]. The IRR for MTB in patients with hematological malignancies (IRR $=3.53$; 95\%CI:1.637.64) was moderately higher compared to patients with solid tumors (IRR $=2.25$; 95\%CI:1.96-2.58).

Taken together, the aforementioned results support screening for LTBC among patients with hematologic malignancies, HNSCC, and lung cancer, based on the substantially increased incidence of active MTB in these groups. However, in patients with other solid tumors, screening for LTBC is not routinely performed and a riskstratified approach should be proposed. First, the risk of MTB in these types of cancer is significantly lower compared to other immunocompromised groups, such as patients with HIV (RR:50-110) [47, 48], contact with individuals with active MTB (RR:10.4) [49], patients with chronic renal failure (RR:7.8) [5] and patients being treated with TNF-a inhibitors (RR:1.8-29.3) [50]. Although, the reactivation of MTB can occur at any time after initial infection [3], the estimated cumulative lifetime risk for developing active MTB is calculated by IRR, and this time-dependent parameter guides decisions about LTBC screening and chemoprophylaxis. For example, in chronic conditions with moderate effect upon life expectancy, such as diabetes and chronic renal failure, the potential risk is expected to last a whole lifetime. In contrast, the short-term immunosuppression induced by adjuvant treatment in earlier cancer stage, and the poor prognosis in metastatic stage, provide a reduced risk for developing active MTB infection. There is not a single threshold-risk of MTB (an IRR cut-off) over which to establish systematic screening and treatment for LTBC, in cancer patients, independent of other risk factors. The potential harms and benefits of LTBC treatment due to drug interactions or toxicities will also need to be weighed on an individual basis [60]. Among patients at low risk for hepatotoxicity (mainly due to isoniazide), LTBC testing is suggested for cases with an expected 5-year survival > 25\% while among patients at increased risk for hepatotoxicity, LTBC testing is suggested for individuals with an expected 5-year survival $>50 \%[10,11]$. All these aspects including the type of cancer, the exposure to mycobacterium, the expected prognosis, the host comorbidities and the possible drug toxicities should be taken into account at the time of consideration for LTBC screening.

\section{Recommended tests for LTBC screening}

Two screening tests for LTBC are currently used: a) the tuberculin skin test (TST), and b) the blood test of interferon-gamma release assay (IGRA). The TST requires intradermal placement of tuberculin purified protein derivative and interpretation of skin erythema and induration response $48-72 \mathrm{~h}$ later (for palpable swelling). Both T-SPOT.TB (Oxford Immunotec Global) and QuantiFERON-TB Gold In-Tube (Qiagen) are currently approved IGRAs and require just a blood sample for results within 8 to $30 \mathrm{~h}$ [51]. Despite this many patient factors and health system parameters may influence the selection of a screening test [61], guidelines by WHO, USPSTF, ATS, CDC and IDSA support testing with IGRA over TST for diagnosis of LTBC in individuals with low-to-intermediate risk of progression to active disease, and either IGRA or TST or dual testing (if first one is negative) at highest risk of developing active MTB $[51,62]$. Before consideration of usage of TNF-a blocking agents, IGRA is preferable to TST because of lower false-positive results in patients treated with corticosteroids and/or with a previous BCG vaccination [63]. However, after reviewing 19 studies in immunosuppressed patients, Hasan et al. found that TST and IGRA were of equal value for LTBC screening [12].

\section{Exclusion of active tuberculosis on a cancer patient}

After a positive IGRA or TST and prior to LTBC treatment, all patients must be evaluated to rule out active tuberculosis and to minimize the risk of drug resistance associated with anti-tuberculosis monotherapy. The evaluation includes clinical history, physical examination, and chest radiograph and respiratory sampling. The exclusion of active MTB infection remains a diagnostic challenge in an oncologic patient, since many symptoms (such as cough $>2$ weeks' duration, fevers, night sweats, weight loss and new interstitial infiltrates) can be due to either cancer progression or infection. Patients with relevant clinical manifestations and/or abnormal chest radiograph should submit 3 sputum specimens (obtained via cough or induction at least $8 \mathrm{~h}$ apart and including at least one earlymorning specimen) for acid-fast bacilli (AFB) smear, mycobacterial culture, and nucleic acid amplification testing. All unexpected suspicious lung lesions depicted by imaging should be investigated or biopsied, if possible. As outlined by two older studies, active tuberculosis occurred, concurrently or soon after the tumor diagnosis, in more than half of patients with HNSCC or lung cancer [64, 65]. This active incidence of MTB remains elevated for the first year after cancer diagnosis and treatment initiation, and after that, declines over time $[56,66]$; for example in hematological malignancies, from 12.01\% (95\%CI:10.81$13.30)$ in the first 6 months, reduces to $2.70 \%$ (95\% CI: 2.12-3.39) after 24 months [66]. Although the initially 
closer follow-up might result in an overestimation, it is clear that the presentation of cancer is a significant factor in the risk for developing active tuberculosis.

\section{Targeted LTBC screening before immunotherapy}

In developing countries with high prevalence of MTB, the limited use of ICBs and the short follow-up may lead to underreporting of the real risk in the current literature, while in developed countries, personalized therapeutic approaches based on the cancer stage, tumor molecular profile and expected prognosis makes it more difficult to differentiate between the risk arising from cancer per se and that arising from a specific treatment option. Although therapeutic advances and prolongation of survival in melanoma patients may influence the lifetime risk of developing or reactivating MTB infection, it is not clear whether the addition of immunotherapy or other anticancer treatments significantly increase the risk associated with cancer itself. A direct comparison of MTB rates between ICB-treated and non-ICB treated cancer patients is not feasible, since available data are limited. To enlighten as much as we can any difference, we present here the population-based tuberculosis rates rather than standardized individual risk for person-years, which is the standard approach. An older report by Memorial Sloan-Kettering Cancer Center described that the incidence of MTB was highest in patients with hematologic malignancies with a notification rate greater than 200 cases per 100,000 persons, $>2 / 1000$ ), a rate similar to that of patients with HIV infection or with recent mycobacterial exposure. The incidence varied significantly according to the country of birth [67]. Among patients with solid tumors, the overall rate of MTB reactivation was 39 cases per 100,000 persons $(0.39 / 1000)$ and varied significantly between US-born and non-USborn patients. Patients with HNSCC had a substantially increased MTB rate (135 cases per 100,000 persons, $1.35 / 1000)$ that was unrelated to country of birth. In this large study, the mortality rate of MTB-infected cancer patients was similarly high (25\%) with this one described here in ICB-treated patients $(28.57 \%)$ and all cases died within 3 months of MTB diagnosis [67]. According to the French prospective registry managed by Gustave Roussy cancer center the rate of tuberculosis among cancer patients receiving anti-PD1/PD-L1 agents was estimated about $1 / 1000$ patients [46]. In our institution we have treated with ICBs, approximately 500 melanoma patients in the adjuvant or metastatic setting, either in a clinical trial or after the regulatory approval of immunotherapy. Among this ICB-treated melanoma population, we diagnosed the 2 above-mentioned cases with MTB reactivation $(\sim 2 / 500)$. With the assumption that our patients had been exposed for the same duration in immunotherapy with those treated at Gustave Roussy, the estimated Poisson rates for the two populations did not differ significantly. Notably, this high observed MTB rate could not be justified only by the WHO and ECDC country-specific data for USA (3.1 per 100,000), France (9 per 100,000), and Greece (4.5 per 100,000), even if one takes into account the underreporting of diagnosed cases, in Greece and the significant immigration from endemic regions (Iraq, Afghanistan, India, Africa etc.).

Given that an anti-PD1 agent might favor tuberculosis reactivation, although without strong and direct evidence, Picchi et al. [46], suggested screening for LTBC with an IGRA in all cancer patients before initiation of immunotherapy. However, the cost of such strategy may not be affordable and the clinical benefit of treating all positive cases remains uncertain [68]. At this point, we propose that a targeted LTBC screening before the administration of ICPIs should be considered, especially in cancer individuals with one or more independent risk factors (diabetes, chronic renal failure, possible exposure to MTB or further immunosuppression). Apart from individual risk factors, all candidates for adjuvant immunotherapy (e.g. cases with melanoma stage III), which are considered free of cancer, should be tested for LTBC to ensure that they will not experience at the close future any complication by reactivated MTB. Since there is no knowledge whether LTBC testing is affected by the ICPIs, it is generally suggested to perform this screening before the administration of immunotherapy. Recently, ESCMID Study Group Consensus supported LTBC screening before starting of any anti-TNF- $\alpha$ therapy as the standard of care, followed by appropriate anti-tuberculosis therapy [69].

Cancer patients treated with immunotherapy develop a continuously expanding spectrum of irAEs, and most of them require steroids and/or anti-TNFa agents for management when these become severe. Based on a recent review of infectious complications in melanoma patients treated with ICBs, the major risk factor for serious infections was the use of additional immunosuppressive agents, reaching an incidence of $13.5 \%$ in patients for corticosteroids or infliximab but only $2 \%$ in those who did not [9]. In agreement with our observations, infections occurred during the first 6 months after initiation of ICBs in 79.6\% of patients [9]. Cancer patients on dual immunotherapy with nivolumab and ipilimumab or on combination with immuno- and chemo-therapy present higher incidence of irAEs (requiring steroids or steroid-sparing agents) and deeper degree of immunomodulation (developing more often ICB-associated infections) [6]. These patients belong in the high-risk group for MTB reactivation and need to be screened for LTBC before initiation of immunotherapy. However, in some cases the critical status of patients due to their disease or their severe irAEs may not permit 
waiting for LTBC testing results and the anticancer treatment is rashly prioritized.

The treatment of LTBC includes 4 months of rifampin or 9 months of isoniazid, or 3 months of once weekly directly observed therapy with isoniazid plus rifapentin [70]. However, no available data exist regarding LTBC chemoprophylaxis in PD-1/PD-L1 blockade and the therapeutic strategy here is based on evidence from TNF-a inhibition. According to findings from patients receiving anti-TNF agents, a 4-week chemoprevention with isoniazid reduces significantly the risk of developing active MTB [17]. Although more recent studies and CDC guidelines have suggested that LTBC treatment can start any time (even concurrent) with anti-TNF-a administration [71, 72], we propose that treatment prior to beginning anti-PD-1/anti-PD-L1 antibodies (e.g. 2 weeks) is more reasonable in order to assure patient tolerance of anti-tuberculosis prophylaxis. Patients should be monitored monthly for clinical signs of hepatitis during therapy for LTBC.

\section{Management of MTB reactivation during immunotherapy}

To date, there is no evidence-based strategy for treating reactivated MTB during immunotherapy. Despite the theoretical benefit of PD-1/PD-L1 blockade in treating various infections, as well as tuberculosis [73, 74], it is generally supported that ICBs should be withheld during active infection, because of the possibility of an exaggerated inflammatory response. However, the exact timing for safe resumption of immunotherapy after initiation of anti-tuberculosis treatment remains to be defined. Adopting the same strategy with LTBC prophylaxis, a 2-weeks interval with anti-tuberculosis treatment is also suggested before re-starting of immunotherapy. In cases with concurrent initiation of anti-tuberculosis treatment and maintenance of anti-PD-1/PD-L1 therapy, close monitoring is required to detect overlapping toxicities, especially liver dysfunction.

\section{Conclusions}

It is accepted that ICBs may have infectious complications, indirectly as a consequence of the need for corticosteroids or TNF-a inhibitors to control irAEs associated with ICB therapy. Tuberculosis may be an exception to this rule, as the majority of reported cases from the literature and our experience were receiving neither corticosteroids nor TNF-a inhibitors when their reactivation was documented. Therefore, MTB reactivation may represent a direct complication of immunotherapy, although more data are needed to unequivocally establish this. The exact mechanism of increased susceptibility to MTB following administration of ICBs is not yet known. Preclinical data recognize a crucial role of PD-1/PD-L1 blocking in $\mathrm{T}$ cell exhaustion, evasion of immune surveillance and development of active tuberculosis. However, in clinical practice, the management of $\mathrm{M}$. tuberculosis among cancer patients receiving ICBs presents challenges. Cancer itself is an independent risk factor for developing active MTB infection. This generally occurs early in the course of disease, and cancer progression is the most common misdiagnosis when constitutional symptoms such as weight loss and fever, common with active MTB, are developed. Thus, before changing treatment for a supposed disease progression or initiating corticosteroids for a suspected irAE, all cancer patients with appropriate symptomatology should be tested for tuberculosis and checked for any previous exposure to MTB, and other risk factors. The prompt diagnosis of a mycobacterial infection, even in a subclinical stage, is essential to avoid later potentially morbid exacerbation. Given that inhibition of PD-1/PDL1 pathway may favor tuberculosis reactivation, targeted screening for LTBC is suggested before initiation of an ICPI, especially in cancer subjects with additional independent risk factors (e.g. host comorbidities, exposure to MTB endemic regions, and immunosuppression). The preferred diagnostic modality (e.g. a single test or combination of TST and IGRA) for LTBC screening in these patients has not been clearly defined. In addition, no available data exist for the management of latent or active tuberculosis during PD-1/PD-L1 blockade; for this reason, therapeutic guidelines are adopted from the management of patients receiving TNF-a inhibition. In general, in case of active tuberculosis, ICPIs are temporarily withheld, any further immunosuppression is discontinued and anti-tuberculosis treatment is timely initiated. Also, in patients diagnosed with either active or latent tuberculosis, it is not clear how long after the corresponding anti-TB treatment ICPIs should be safely resumed or initiated, with a duration of 2-4 weeks to be suggested. The continuously expanded implementation of IPCIs in cancer treatment requires resolving of these challenges by upcoming research data in order to maximize the clinical benefits of immunotherapy uninterruptedly and safely.

\section{Abbreviations \\ AFB: Acid-fast bacilli; ATS: American Thoracic Society; BAL: Bronchoalveolar lavage; CDC: Center for Disease Control and Prevention; CRP: C reactive protein; CT: Computed tomography; HNSCC: Head and neck squamous cell carcinoma; ICBs: Immune checkpoint inhibitors; IDSA: Infectious Diseases Society of America; IFN: Interferon; irAEs: Immune-related adverse events; IRIS: Immune reconstitution inflammatory syndrome; IRR: Incidence rate ratio; LTBC: Latent tuberculosis infection; MTB: Mycobacterial tuberculosis; NICE: National Institute for Health and Care Excellence; NSCLC: Non-small cell lung cancer; PCR: Polymerase chain reaction; Th1: T-helper type 1; TNF- a: Tumor necrosis factor-alpha; TST: Tuberculin skin test; USPSTF: US Preventive Services Task Force; WHO: World Health Organization}

\section{Acknowledgements}

Not applicable.

\section{Authors' contributions}

AA treated both presented cases; AA and DZ reviewed the literature; DZ, MS JK and HG wrote the manuscript. All authors edited, read and approved the final manuscript. 


\section{Funding}

No funding supported the writing and the submission of this study.

\section{Availability of data and materials}

Data supporting the recommendations of this article are included within the reference list. Please contact corresponding author for any further data request or supplementary information.

\section{Ethics approval and consent to participate}

In accordance with the ethical declaration of Helsinki (1964) and its later amendments, the relatives of the first patient and the second patient gave written informed consent prior to reporting of their cases.

\section{Consent for publication}

The relatives of the first patient and the second patient gave a written consent for publication on a local form approved by the Medical Ethics Committee of our institution.

\section{Competing interests}

HG has received grants and personal fees by Roche, BMS, MSD, Novartis and personal fees by Amgen and Pierre Fabre, outside the submitted work; JK has received personal fees for advisory/consultancy by BMS, Immunocore, lovance, Novartis, Elsevier and research grant by BMS, Checkmate, Immunocore, outside the submitted work. All other authors declare no conflict of interest.

\section{Author details}

'First Department of Medicine, Laiko General Hospital, National and Kapodistrian University of Athens School of Medicine, 11527 Athens, Greece. ${ }^{2}$ Division of Hematology/Oncology, University of Pittsburgh School of Medicine, Pittsburgh, PA 15213, USA.

Received: 10 May 2019 Accepted: 23 August 2019 Published online: 04 September 2019

\section{References}

1. Wei SC, Duffy CR, Allison JP. Fundamental mechanisms of immune checkpoint blockade therapy. Cancer Discov. 2018:8:1069-86.

2. Mellman I, Coukos G, Dranoff G. Cancer immunotherapy comes of age. Nature. 2011:480:480-9.

3. Schreiber RD, Old LJ, Smyth MJ. Cancer immunoediting: integrating immunity's roles in cancer suppression and promotion. Science. 2011;331: 1565-70.

4. De Velasco G, Je Y, Bosse $D$, et al. Comprehensive meta-analysis of key immune-related adverse events from CTLA-4 and PD-1/PD-L1 inhibitors in Cancer patients. Cancer Immunol Res. 2017;5:312-8.

5. Heinzerling L, Ascierto PA, Dummer R, et al. Adverse events 2.0-Let us get SERIOs: New reporting for adverse event outcomes needed in the era of immunooncology. Eur J Cancer. 2019:112:29-31.

6. Haanen J, Carbonnel F, Robert C, et al. Management of toxicities from immunotherapy: ESMO Clinical Practice Guidelines for diagnosis, treatment and follow-up. Ann Oncol. 2018;29:iv264-iv66.

7. Kyi C, Hellmann MD, Wolchok JD, et al. Opportunistic infections in patients treated with immunotherapy for cancer. J Immunother Cancer. 2014;2:19.

8. Fishman JA, Hogan JI, Maus MV. Inflammatory and Infectious Syndromes Associated with Cancer Immunotherapies. Clin Infect Dis. 2018. https://doi. org/10.1093/cid/ciy 1025 .

9. Del Castillo M, Romero FA, Arguello E, et al. The Spectrum of serious infections among patients receiving immune checkpoint blockade for the treatment of melanoma. Clin Infect Dis. 2016:63:1490-3.

10. Dobler CC, Cheung K, Nguyen J, et al. Risk of tuberculosis in patients with solid cancers and haematological malignancies: a systematic review and meta-analysis. Eur Respir J. 2017;50. https://doi.org/10.1183/13993003.0015 7-2017.

11. Cheng MP, Abou Chakra CN, Yansouni CP, et al. Risk of active tuberculosis in patients with Cancer: a systematic review and meta-analysis. Clin Infect Dis. 2017:64:635-44.

12. Hasan $T, A u E, C h e n ~ S$, et al. Screening and prevention for latent tuberculosis in immunosuppressed patients at risk for tuberculosis: a systematic review of clinical practice guidelines. BMJ Open. 2018;8:e022445.

13. Dobler CC, McDonald SP, Marks GB. Risk of tuberculosis in dialysis patients: a nationwide cohort study. PLoS One. 2011;6:e29563.
14. Aguado JM, Torre-Cisneros J, Fortun J, et al. Tuberculosis in solid-organ transplant recipients: consensus statement of the group for the study of infection in transplant recipients (GESITRA) of the Spanish Society of Infectious Diseases and Clinical Microbiology. Clin Infect Dis. 2009;48:1276-84.

15. Budak-Alpdogan T, Tangun Y, Kalayoglu-Besisik $S$, et al. The frequency of tuberculosis in adult allogeneic stem cell transplant recipients in Turkey. Biol Blood Marrow Transplant. 2000;6:370-4.

16. Askling J, Fored CM, Brandt $L$, et al. Risk and case characteristics of tuberculosis in rheumatoid arthritis associated with tumor necrosis factor antagonists in Sweden. Arthritis Rheum. 2005;52:1986-92.

17. Carmona L, Gomez-Reino JJ, Rodriguez-Valverde V, et al. Effectiveness of recommendations to prevent reactivation of latent tuberculosis infection in patients treated with tumor necrosis factor antagonists. Arthritis Rheum. 2005;52:1766-72.

18. Tubach F, Salmon D, Ravaud P, et al. Risk of tuberculosis is higher with antitumor necrosis factor monoclonal antibody therapy than with soluble tumor necrosis factor receptor therapy: the three-year prospective French research axed on tolerance of biotherapies registry. Arthritis Rheum. 2009;60:1884-94.

19. Wolfe F, Michaud $K$, Anderson J, et al. Tuberculosis infection in patients with rheumatoid arthritis and the effect of infliximab therapy. Arthritis Rheum. 2004:50:372-9

20. Getahun H, Gunneberg C, Granich R, et al. HIV infection-associated tuberculosis: the epidemiology and the response. Clin Infect Dis. 2010; 50(Suppl 3):S201-7.

21. Min J, Kwon SK, Jeong HW, et al. End-stage renal disease and risk of active tuberculosis: a Nationwide population-based cohort study. J Korean Med Sci. 2018;33:e341.

22. Dooley KE, Chaisson RE. Tuberculosis and diabetes mellitus: convergence of two epidemics. Lancet Infect Dis. 2009;9:737-46.

23. Christopoulos A, Saif MW, Sarris EG, et al. Epidemiology of active tuberculosis in lung cancer patients: a systematic review. Clin Respir J. 2014;8:375-81.

24. Wherry EJ. T cell exhaustion. Nat Immunol. 2011;12:492-9.

25. Lazar-Molnar E, Chen B, Sweeney KA, et al. Programmed death-1 (PD-1)deficient mice are extraordinarily sensitive to tuberculosis. Proc Natl Acad Sci U S A. 2010;107:13402-7.

26. Barber DL, Mayer-Barber KD, Feng CG, et al. CD4 T cells promote rather than control tuberculosis in the absence of PD-1-mediated inhibition. J Immunol. 2011:186:1598-607.

27. Tousif S, Singh Y, Prasad DV, et al. T cells from programmed Death-1 deficient mice respond poorly to Mycobacterium tuberculosis infection. PLoS One. 2011;6:e19864.

28. Sakai S, Kauffman KD, Sallin MA, et al. CD4 T cell-derived IFN-gamma plays a minimal role in control of pulmonary Mycobacterium tuberculosis infection and must be actively repressed by PD-1 to prevent lethal disease. PLoS Pathog. 2016;12:e1005667.

29. Trinath J, Maddur MS, Kaveri SV, et al. Mycobacterium tuberculosis promotes regulatory T-cell expansion via induction of programmed death-1 ligand 1 (PD-L1, CD274) on dendritic cells. J Infect Dis. 2012;205:694-6.

30. Versteven M, Van den Bergh JMJ, Marcq E, et al. Dendritic cells and programmed Death-1 blockade: a joint venture to combat Cancer. Front Immunol. 2018:9:394.

31. Benson DM Jr, Bakan CE, Mishra A, et al. The PD-1/PD-L1 axis modulates the natural killer cell versus multiple myeloma effect: a therapeutic target for CT-011, a novel monoclonal anti-PD-1 antibody. Blood. 2010;116:2286-94.

32. Alvarez IB, Pasquinelli $V$, Jurado JO, et al. Role played by the programmed death-1-programmed death ligand pathway during innate immunity against Mycobacterium tuberculosis. J Infect Dis. 2010;202:524-32.

33. Norris $\mathrm{S}$, Coleman $\mathrm{A}$, Kuri-Cervantes $\mathrm{L}$, et al. PD-1 expression on natural killer cells and CD8(+) T cells during chronic HIV-1 infection. Viral Immunol. 2012:25:329-32.

34. Cao S, Li J, Lu J, et al. Mycobacterium tuberculosis antigens repress Th1 immune response suppression and promotes lung cancer metastasis through PD-1/PDI-1 signaling pathway. Cell Death Dis. 2019;10:44.

35. Shen L, Gao Y, Liu Y, et al. PD-1/PD-L pathway inhibits M.tb-specific CD4(+) T-cell functions and phagocytosis of macrophages in active tuberculosis. Sci Rep. 2016;6:38362.

36. Shen L, Shi H, Gao Y, et al. The characteristic profiles of PD-1 and PD-L1 expressions and dynamic changes during treatment in active tuberculosis. Tuberculosis (Edinb). 2016:101:146-50.

37. Barber DL, Sakai S, Kudchadkar RR, et al. Tuberculosis following PD-1 blockade for cancer immunotherapy. Sci Transl Med. 2019;11. https://doi. org/10.1126/scitransImed.aat2702. 
38. Meintjes G, Stek C, Blumenthal L, et al. Prednisone for the prevention of paradoxical tuberculosis-associated IRIS. N Engl J Med. 2018;379:1915-25.

39. Rao M, Ligeiro D, Maeurer M. Precision medicine in the clinical management of respiratory tract infections including multidrug-resistant tuberculosis: learning from innovations in immuno-oncology. Curr Opin Pulm Med. 2019;25:233-41

40. Rao M, Ippolito G, Mfinanga S, et al. Improving treatment outcomes for MDR-TB - novel host-directed therapies and personalised medicine of the future. Int J Infect Dis. 2019;80S:S62-7.

41. Lee JJ, Chan A, Tang T. Tuberculosis reactivation in a patient receiving antiprogrammed death-1 (PD-1) inhibitor for relapsed Hodgkin's lymphoma. Acta Oncol. 2016;55:519-20.

42. Fujita K, Terashima T, Mio T. Anti-PD1 antibody treatment and the development of acute pulmonary tuberculosis. J Thorac Oncol. 2016;11: 2238-40.

43. Chu YC, Fang KC, Chen HC, et al. Pericardial tamponade caused by a hypersensitivity response to tuberculosis reactivation after anti-PD-1 treatment in a patient with advanced pulmonary adenocarcinoma. J Thorac Oncol. 2017;12:e111-e14

44. He W, Zhang X, Li W, et al. Activated pulmonary tuberculosis in a patient with melanoma during PD-1 inhibition: a case report. Onco Targets Ther. 2018;11:7423-7.

45. Jensen $\mathrm{KH}$, Persson $\mathrm{G}$, Bondgaard $\mathrm{AL}$, et al. Development of pulmonary tuberculosis following treatment with anti-PD-1 for non-small cell lung cancer. Acta Oncol. 2018;57:1127-8.

46. Picchi H, Mateus C, Chouaid C, et al. Infectious complications associated with the use of immune checkpoint inhibitors in oncology: reactivation of tuberculosis after anti PD-1 treatment. Clin Microbiol Infect. 2018;24:216-8.

47. Elkington PT, Bateman AC, Thomas GJ, et al. Implications of tuberculosis reactivation after immune checkpoint inhibition. Am J Respir Crit Care Med. 2018;198:1451-3.

48. Tsai CC, Chen JH, Wang YC, et al. Re-activation of pulmonary tuberculosis during anti-programmed death-1 (PD-1) treatment. QJM. 2019;112:41-2.

49. Takata S, Koh G, Han Y, et al. Paradoxical response in a patient with nonsmall cell lung cancer who received nivolumab followed by antiMycobacterium tuberculosis agents. J Infect Chemother. 2019;25:54-8.

50. Latent tuberculosis infection: updated and consolidated guidelines for programmatic management. Geneva: World Health Organization 2018.

51. Force USPST, Bibbins-Domingo K, Grossman DC, et al. Screening for latent tuberculosis infection in adults: US preventive services task Force recommendation statement. JAMA. 2016;316:962-9.

52. Targeted tuberculin testing and treatment of latent tuberculosis infection. This official statement of the American Thoracic Society was adopted by the ATS Board of Directors, July 1999. This is a Joint Statement of the American Thoracic Society (ATS) and the Centers for Disease Control and Prevention (CDC). This statement was endorsed by the Council of the Infectious Diseases Society of America. (IDSA), September 1999, and the sections of this statement. Am J Respir Crit Care Med. 2000;161:S221-47.

53. Morrison VA. Immunosuppression associated with novel chemotherapy agents and monoclonal antibodies. Clin Infect Dis. 2014;59(Suppl 5):S360-4.

54. Cognetti DM, Weber RS, Lai SY. Head and neck cancer: an evolving treatment paradigm. Cancer. 2008;113:1911-32.

55. National Institute for Health and Care Excellence (2016) Tuberculosis (NICE Guideline 33). Available at: https://www.nice.org.uk/guidance/ng33/ resources/tuberculosis-1837390683589. Accessed 22 Apr 2019

56. Simonsen DF, Farkas DK, Horsburgh CR, et al. Increased risk of active tuberculosis after cancer diagnosis. J Inf Secur. 2017;74:590-8.

57. Soh AZ, Chee CBE, Wang YT, et al. Alcohol drinking and cigarette smoking in relation to risk of active tuberculosis: prospective cohort study. BMJ Open Respir Res. 2017:4:e000247.

58. Scholmerich J. Postgastrectomy syndromes--diagnosis and treatment. Best Pract Res Clin Gastroenterol. 2004;18:917-33.

59. Dai G, Phalen S, McMurray DN. Nutritional modulation of host responses to mycobacteria. Front Biosci. 1998;3:e110-22.

60. Getahun H, Matteelli A, Abubakar I, et al. Management of latent Mycobacterium tuberculosis infection: WHO guidelines for low tuberculosis burden countries. Eur Respir J. 2015:46:1563-76.

61. Collins LF, Geadas C, Ellner JJ. Diagnosis of latent tuberculosis infection: too soon to pull the plug on the tuberculin skin test. Ann Intern Med. 2016;164: $122-4$.
62. Lewinsohn DM, Leonard MK, LoBue PA, et al. Official American Thoracic Society/Infectious Diseases Society of America/Centers for Disease Contro and Prevention clinical practice guidelines: diagnosis of tuberculosis in adults and children. Clin Infect Dis. 2017;64:111-5.

63. Ruan $Q$, Zhang $S, A i$ J, et al. Screening of latent tuberculosis infection by interferon-gamma release assays in rheumatic patients: a systemic review and meta-analysis. Clin Rheumatol. 2016;35:417-25.

64. Kaplan MH, Armstrong D, Rosen P. Tuberculosis complicating neoplastic disease. A review of 201 cases. Cancer. 1974;33:850-8.

65. Libshitz HI, Pannu HK, Elting LS, et al. Tuberculosis in cancer patients: an update. J Thorac Imaging. 1997;12:41-6.

66. Seo GH, Kim MJ, Seo S, et al. Cancer-specific incidence rates of tuberculosis: A 5-year nationwide population-based study in a country with an intermediate tuberculosis burden. Medicine (Baltimore). 2016;95:e4919.

67. Kamboj M, Sepkowitz KA. The risk of tuberculosis in patients with cancer. Clin Infect Dis. 2006:42:1592-5.

68. Tasillo A, Salomon JA, Trikalinos TA, et al. Cost-effectiveness of testing and treatment for latent tuberculosis infection in residents born outside the United States with and without medical comorbidities in a simulation model. JAMA Intern Med. 2017;177:1755-64.

69. Baddley JW, Cantini F, Goletti D, et al. ESCMID study Group for Infections in compromised hosts $(\mathrm{ESGICH})$ consensus document on the safety of targeted and biological therapies: an infectious diseases perspective (soluble immune effector molecules [1]: anti-tumor necrosis factor-alpha agents). Clin Microbiol Infect. 2018;24(Suppl 2):S10-20.

70. Menzies D, Adjobimey M, Ruslami R, et al. Four months of rifampin or nine months of isoniazid for latent tuberculosis in adults. N Engl J Med. 2018;379: 440-53.

71. Winthrop KL, Siegel JN, Jereb J, et al. Tuberculosis associated with therapy against tumor necrosis factor alpha. Arthritis Rheum. 2005;52:2968-74.

72. Hsia EC, Schluger N, Cush JJ, et al. Interferon-gamma release assay versus tuberculin skin test prior to treatment with golimumab, a human antitumor necrosis factor antibody, in patients with rheumatoid arthritis, psoriatic arthritis, or ankylosing spondylitis. Arthritis Rheum. 2012;64:2068-77.

73. Dyck L, Mills KHG. Immune checkpoints and their inhibition in cancer and infectious diseases. Eur J Immunol. 2017:47:765-79.

74. Jurado JO, Alvarez IB, Pasquinelli V, et al. Programmed death (PD)-1:PDligand 1/PD-ligand 2 pathway inhibits $T$ cell effector functions during human tuberculosis. J Immunol. 2008;181:116-25.

\section{Publisher's Note}

Springer Nature remains neutral with regard to jurisdictional claims in published maps and institutional affiliations.

Ready to submit your research? Choose BMC and benefit from:

- fast, convenient online submission

- thorough peer review by experienced researchers in your field

- rapid publication on acceptance

- support for research data, including large and complex data types

- gold Open Access which fosters wider collaboration and increased citations

- maximum visibility for your research: over $100 \mathrm{M}$ website views per year

At $\mathrm{BMC}$, research is always in progress.

Learn more biomedcentral.com/submission 